\title{
Quantitative image analysis of white etching areas in SAE52100 bearing steel
}

\author{
Philippe T. Pinard ${ }^{1}$, Mehmet Özel ${ }^{2}$, Moritz Ploß ${ }^{3}$ and Silvia Richter ${ }^{1}$ \\ ${ }^{1}$ Central Facility for Electron Microscopy, RWTH Aachen University, Aachen, Germany \\ ${ }^{2}$ Institute for Machine Elements and Machine Design, RWTH Aachen University, Aachen, Germany \\ ${ }^{3}$ Institute for Materials Applications in Mechanical Eng, RWTH Aachen University, Aachen, Germany
}

SAE 52100 bearing steel is a common steel grade for several types of bearings used in various applications such as automotive and wind turbines. It consists of a bainitic or martensitic matrix with 0.1 to $5 \mu \mathrm{m}$ dia. spherical carbides. This material suffers from an unexpected failure mechanism where the bearing abruptly fails as early as $10 \%$ of its expected fatigue life [1]. The failure is associated with the formation of white etching areas (WEA) and white etching cracks (WEC) (named after their appearance after Nital etching) within the first millimeter below the contact surface. Different theories exist to explain these microstructural changes: hydrogen induced, carbide dissolution, initiation at inclusions, etc. To better understand the role of the operating conditions (loading, frequency, temperature, type of lubricants, etc.) on the WEA formation, a project was started with the aims of (1) reproducing bearing failures seen in the field within a laboratory-controlled environment, (2) correlating the observed microstructure to the operating conditions and ultimately (3) explaining the failure mechanism.

For the characterization part, it was clear from the beginning that many samples and large areas would need to be observed. The established strategy was to section the failed bearing where pitting or scuffing appears on the contact surface, locate cracks and WEAs using a light optical microscope after polishing and Nital etching, slightly re-polish the surface to remove the etching, and insert the sample in a scanning electron microscope for high resolution images and chemical information. As WEAs can be quite small, the acquisition of electron images was required to ensure an unbiased identification. To facilitate the acquisition, a program was developed to first acquire low magnification images over a large area, detect cracks and then automatically acquire high magnification images along the cracks. A mosaic image resulting from this acquisition is shown in Figure 1.

The next step was to identify the WEAs from the surrounding matrix and cracks using an image analysis routine. The goal is to statistically compare the WEAs found in different cross-sections based on potential criteria such as their size, shape, quantity, distance from the surface, etc. The developed image analysis routine utilized the fact that WEAs contain very small grains [2] and therefore appear brighter in a backscattered electron image due to lower electron channelling. The image analysis methodology goes as follows (Figure 2): thresholding to identify the cracks (low intensity pixels), morphological dilation of the identified cracks to remove edge effects (topographical effects), removal of the cracks from the original image, median filter to remove noise, smoothing to remove intensity fluctuations in the matrix, thresholding of WEAs using a random-walk algorithm and finally removal of false positive WEAs based on the criterion that WEAs should be close to a crack. All image processing procedures were written in Python with the scikit-image library [3].

Chemical information of the WEAs and their surroundings was obtained using a field emission electron microprobe (JEOL JXA8530F). This instrument was also used for the image acquisition. As indicated in [4], quantification of the carbon concentration in SAE52100 is challenging due to the high concentration of chromium. As preliminary results, a lower carbon concentration was observed in the WEAs than in the surrounding matrix (Figure 3). Further analysis of the composition as well as examples of failed bearings under different operating conditions will be presented. 
[1] Evans et al, Tribology International 65 (2013), pp. 146-160.

[2] Kang et al, Scripta Materialia 69 (2013), pp. 630-633.

[3] van der Walt et al, PeerJ 2:e453 (2014)

[4] Pinard and Richter, IOP Conf. Series: Materials Science and Engineering 55 (2014), p. 012016.

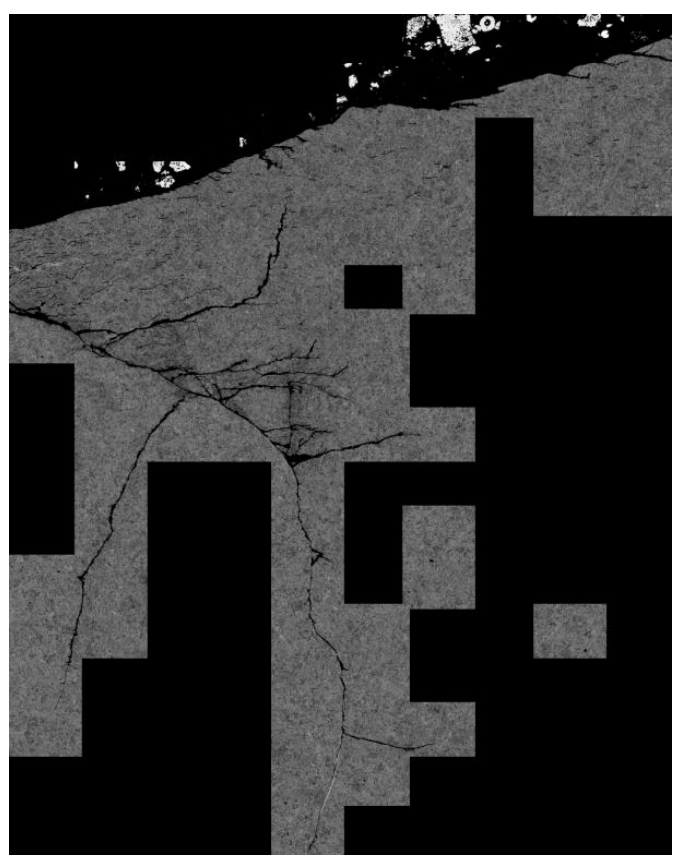

Figure 1: Result of the automated acquisition of high resolution images along the cracks.

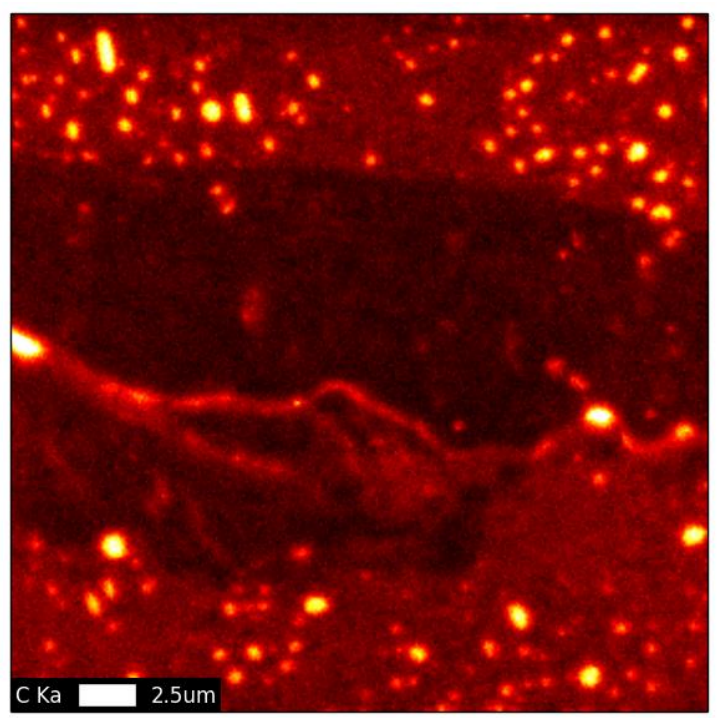

(a)

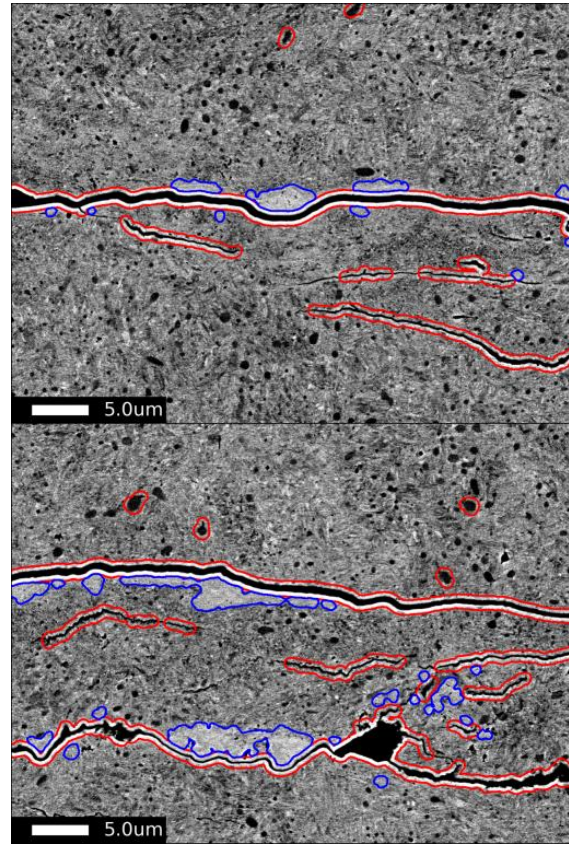

Figure 2: Examples of the image analysis routine used to automatically detect cracks (red) and white etching areas (blue).

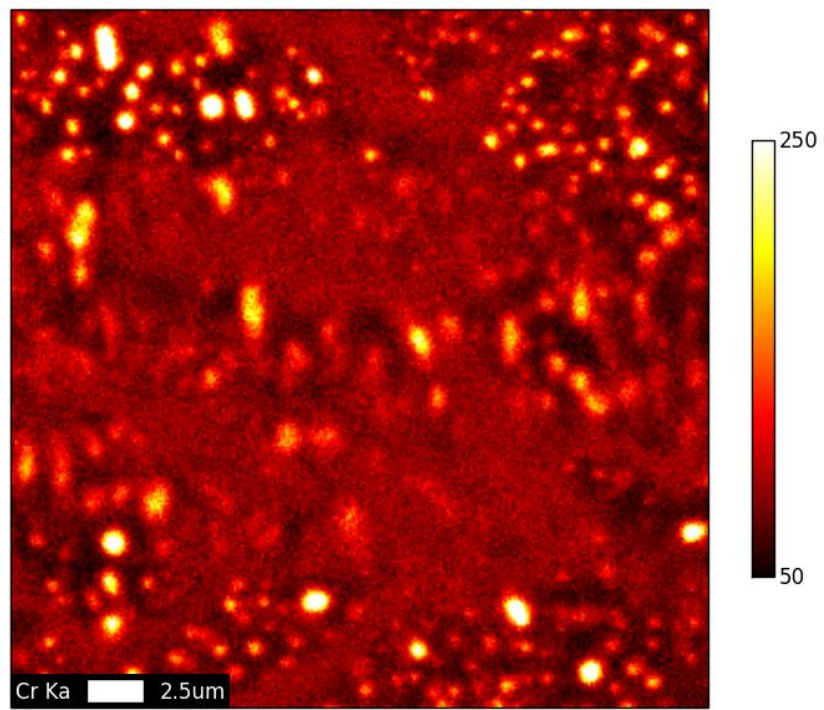

(b)

Figure 3: Qualitative carbon (a) and chromium (b) mapping of a white etching area. 